\title{
THE CLASSIFICATION PROBLEM FOR COMPACT COMPUTABLE METRIC SPACES
}

\author{
ALEXANDER G. MELNIKOV AND ANDRÉ NIES
}

\begin{abstract}
We adjust methods of computable model theory to effective analysis. We use index sets and infinitary logic to obtain classificationtype results for compact computable metric spaces. We show that every compact computable metric space can be uniquely described, up to an isomorphism, by a computable $\Pi_{3}$ formula, and that orbits of elements are uniformly given by computable $\Pi_{2}$ formulas. We show that the index set for such spaces is $\Pi_{3}^{0}$-complete, and the isomorphism problem is $\Pi_{2}^{0}$-complete within its index set. We also give further classification results for special classes of compact spaces, and for other related classes of Polish spaces. Finally, as our main result we show that each compact computable metric space is $\Delta_{3}^{0}$-categorical, and there exists a compact computable Polish space which is not $\Delta_{2}^{0}$-categorical.
\end{abstract}

\section{INTRODUCTION}

An equivalence relation on a standard Borel space is called smooth if it is Borel reducible to the equality relation on $\mathbb{R}$. By a result of Gromov (see [11, proof of 14.2.1]), the isomorphism relation on compact metric spaces is smooth. Thus, every compact metric space can be uniquely described, up to an isomorphism, by a single real. In invariant descriptive set theory, a smooth equivalence relation $E$ is considered trivial: by Silver's theorem, either $E$ is Borel equivalent to equality on $\mathbb{R}$, or $E$ has only countably many classes.

Recall that every compact metric space is separable and complete. Separable complete metric spaces occurring in mathematical practice are usually computable. For instance, $[0,1]^{n}$, the Hilbert cube, $\ell_{2}, \mathcal{C}[0,1]$, and the Urysohn space are computable with any of the standard metrics $[18,16]$. While computable analysis $[18,5,24]$ at present does not have suitable tools to study classification problems, such tools are available in effective algebra and computable model theory $[2,9]$. In this paper, we adapt these methods to computable analysis in order to obtain classification results for compact computable metric spaces.

In contrast to computable analysis, the main objects of computable algebra are countable algebraic structures. These are structures with domain $\mathbb{N}$ and in which the basic operations can be represented by computable functions on $\mathbb{N}$. There are several approaches to classification problems in

We are thankful to Isaac Newton Institute for Mathematical Sciences and, more specifically, Semantics and Syntax: A Legacy of Alan Turing program, for partial support of our project. The first author thanks the Marsden Fund of New Zealand for its support. The second author's research was also partially supported by SPMS, Nanyang Technological University, Singapore. 
computable model theory and effective algebra (see, e.g., $[12,13,6,7])$. We focus on three approaches which use index sets, infinitary computable logic, and the complexity of isomorphisms, respectively. These three approaches are closely related.

Index sets and isomorphism problems. The first approach uses the fact that all partial computable functions can be effectively listed. As a consequence, there exists an effective listing of all partial computable algebraic structures $\left(\mathcal{A}_{e}\right)_{e \in \mathbb{N}}$ which includes all infinite computable algebras. For a class $\mathcal{K}$ of computable algebras, the difficulty of the classification problems is reflected in the following sets:

1. the index set $I_{\mathcal{K}}=\left\{e: \mathcal{A}_{e} \in \mathcal{K}\right\}$ of $\mathcal{K}$, and

2. the isomorphism problem $E_{\mathcal{K}}=\left\{(e, j) \in I_{\mathcal{K}}^{2}: \mathcal{A}_{e} \cong \mathcal{A}_{j}\right\}$ for $\mathcal{K}$.

The complexity of the index sets is measured using the arithmetical, hyperarithmetical, and analytical hierarchies [2]. Recall that the arithmetical hierarchy is defined via iterating quantifiers over computable predicates, and the hyprarithmetical hierarchy extends the arithmetical hierarchy to computable ordinals. Deciding if two algebras from $\mathcal{K}$ are isomorphic might be simpler than detecting whether an algebra belongs to this class. In this case one usually considers the complexity of $E_{\mathcal{K}}$ within $I_{\mathcal{K}}$. For example, $E_{\mathcal{K}}$ is $\Pi_{2}^{0}$ within a $\Pi_{3}^{0}$ set $I_{\mathcal{K}}$ if there exists a $\Pi_{2}^{0}$ set $S \subset \mathbb{N}^{2}$ such that $E_{\mathcal{K}}=S \cap\left(I_{\mathcal{K}} \times I_{\mathcal{K}}\right)$.

A collection of computable models $\mathcal{K}$ is called classifiable if both $I_{\mathcal{K}}$ and $E_{\mathcal{K}}$ are hyperarithmetical. (Usually $\mathcal{K}$ will be closed under isomorphism on computable models.) The position of $I_{\mathcal{K}}$ and $E_{\mathcal{K}}$ in the (hyper)arithmetical hierarchy measures the complexity of the classification. See $[12,13,6,7]$ for further background and results in this direction.

Infinitary computable logic. Ash [1] introduced computable infinitary formulas in the context of computable algebras. An infinitary computable language extends a first-order language by allowing infinite conjunctions and disjunctions over computably enumerable families of formulas. The definition $[1,2]$ uses a recursion scheme. Computable formulas have proved to be of a great importance in computable algebra; see the book of Ash and Knight [2]. We say that a class $\mathcal{K}$ of computable structures closed under isomorphism admits a syntactic description, if there exists a computable infinitary sentence $\Phi$ such that, for any computable $M$, we have $M \models \Phi$ if and only if $M \in \mathcal{K}$. Note that this condition implies that the index set is hyperarithmetical [12]. The converse is known without the restriction to indices for computable structures. Vanden Boom [23] has shown that every hyperarithmetical invariant class can be described by a computable sentence. There is also a syntactic counterpart of requiring that $E_{\mathcal{K}}$ is hyperarithmetical.

Definition 1.1. We say that a class $\mathcal{K}$ of computable structures admits a syntactic classification if there is a hyperarithmetical bound on the complexity of infinitary formulas which describe the orbits of tuples of elements in $M \in \mathcal{K}$ under the action of the automorphism group of $M$.

The complexity of the formulas describing the orbits measures the difficulty of the classification. 
Higher categoricity. The third approach is based on the tradition going back to Fröhlich and Shepherdson [10], Rabin [19], and Mal'cev [14].

Definition 1.2. $[10,19,14]$ A computable algebraic structure $\mathcal{A}$ is computably categorical if every computable algebraic structure isomorphic to $\mathcal{A}$ is in fact computably isomorphic to it.

The notion of computable categoricity can be relativized either fully or partially. For instance, we say that a structure $\mathcal{A}$ is $\Delta_{n}^{0}$-categorical if every computable algebraic structure isomorphic to $\mathcal{A}$ is $0^{(n-1)}$-isomorphic to it. Here $0^{(n-1)}$ stands for the $(n-1)$ 'th iteration of the halting problem. The definition can be pushed to computable ordinals $\alpha$ via a transfinite iteration of the jump.

Various results on $\Delta_{\alpha}^{0}$-categorical structures (see $[15,3,4,17,8]$ ) give evidence that $\Delta_{\alpha}^{0}$-categoricity can be used to measure the complexity of strictures in a class. Most typically, a computable algebraic structure is computably categorical if it either has a "finite basis" (vector spaces, Boolean algebras, abelian groups, etc.), or is highly homogeneous (random graph, dense linear order). If all structures in a class $\mathcal{K}$ are $\Delta_{n}^{0}$-categorical and the index set $I_{\mathcal{K}}$ is at most $\Sigma_{n+2}^{0}$, then the isomorphism problem $E_{\mathcal{K}}$ is at most $\Sigma_{n+2}^{0}$. Also, here are syntactical characterizations of $\Delta_{\alpha}^{0}$ and relatively $\Delta_{\alpha^{-}}^{0}$ categorical structures (see, e.g., [2]) which provide a link to the syntactical approach. If for a class $\mathcal{K}$ there is a computable ordinal $\alpha$ such that all members of $\mathcal{K}$ are $\Delta_{\alpha}^{0}$-categorical, then the class is considered simple. The isomorphism type of $\alpha$ measures the complexity of the class.

1.1. Computable metric spaces. To adjust the effective classification methods to computable analysis, we need some basic definitions. Following the tradition rooted in the works of Turing [21, 22], we say that a real $x$ is computable if for each $k$ we can compute a rational within $2^{-k}$ of $x$.

Definition $1.3([5,18])$. Let $(M, d)$ be a complete separable metric space, and let $\left(q_{i}\right)_{i \in \mathbb{N}}$ be a dense sequence of points in $M$ without repetitions. The triple

$$
\mathcal{M}=\left(M, d,\left(q_{i}\right)_{i \in \mathbb{N}}\right)
$$

is a computable metric space if $d\left(q_{i}, q_{k}\right)$ is a computable real uniformly in $i, k$. We say that $\left(q_{i}\right)_{i \in \mathbb{N}}$ is a computable structure on $M$, and that the $q_{i}$ are the special points of $\mathcal{M}$. A Cauchy name for $x$ is a sequence $\left(r_{p}\right)_{p \in \mathbb{N}}$ of special points converging rapidly to $x$ in the sense that $d\left(r_{p}, r_{p+1}\right)<2^{-p}$.

The general philosophy of effective mathematics is that effectively presented objects should be considered up to effective isomorphisms.

Definition 1.4. [18] An isometry $\Phi$ from $\left(M, d,\left(q_{i}\right)_{i \in \mathbb{N}}\right)$ to $\left(M_{1}, d_{1},\left(p_{i}\right)_{i \in \mathbb{N}}\right)$ is computable if there is an effective procedure which, on input $i$, enumerates a Cauchy name for $\Phi(x)$.

The definition above can be relativized to an oracle $X$ by replacing "effective procedure" by "procedure effective relative to $X$ " in the definition above. That is, we can produce a Cauchy name for $\Phi(x)$ with the help of the oracle. The analog of computable categoricity in the context of separable metric spaces is: 
Definition 1.5. [16] A computable Polish space $(M, d)$ is $\Delta_{n}^{0}$-categorical if for every two computable structures $\left(x_{i}\right)_{i \in \mathbb{N}}$ and $\left(y_{j}\right)_{j \in \mathbb{N}}$ on $M$ there exists a surjective isometry

$$
U:\left(M, d,\left(x_{i}\right)_{i \in \mathbb{N}}\right) \rightarrow\left(M, d,\left(y_{j}\right)_{j \in \mathbb{N}}\right)
$$

which is computable relative to $0^{(n-1)}$.

1.2. Results. We introduce computable infinitary formulas in the context of computable metric spaces (see preliminaries). Our first result is:

Theorem 3.1.

(i) Within the class of computable Polish spaces, each compact member is uniquely described by a computable $\Pi_{3}$ axiom.

(ii) The orbits of special elements in a compact computable Polish space (under the action of its automorphism group) are given uniformly by computable $\Pi_{2}$ formulas.

As a consequence, compact computable Polish spaces admit a syntactic characterization. We apply Theorem 3.1 to show that the collection of compact computable metric spaces is classifiable in the sense given above.

\section{Teorem 4.3.}

(i) The index set CSp of compact computable Polish spaces is $\Pi_{3}^{0}$-complete.

(ii) The isomorphism problem for compact computable Polish spaces is $\Pi_{2}^{0}$-complete within $\Pi_{3}^{0}$.

We also investigate the complexity of index sets of locally compact and connected computable Polish spaces. In Proposition 4.2 we show that their index sets are $\Pi_{1}^{1}$-complete and $\Pi_{1}^{1}$-hard, respectively.

Our main result is the following theorem.

Theorem 5.2. Each compact computable metric space is $\Delta_{3}^{0}$-categorical.

In fact, the proof of Theorem 5.2 can be easily modified to improve $\Delta_{3}^{0}$ upper bound to low relative to $0^{\prime}$. That is, every pair of isomorphic compact computable metric spaces are isomorphic relative to some $S$ such that $S^{\prime}=$ $0^{\prime \prime}$ (see Theorem 5.3). Nonetheless, the upper bound from Theorem 5.2 can not be further improved to $\Delta_{2}^{0}$. In Theorem 5.5 we construct an example of a compact metric space which is not $\Delta_{2}^{0}$-categorical.

\section{Preliminaries}

We view a metric space $(X, d)$ as a structure in the signature

$$
\mathcal{S}=\left\{R_{<q}, R_{>q}: q \in \mathbb{Q}^{+}\right\},
$$

where $R_{<q}$ and $R_{>q}$ are binary relation symbols. The intended meaning of $R_{<q}(x, y)$ is that $d(x, y)<q$. The intended meaning of $R_{>q}(x, y)$ is that $d(x, y)>q$. We denote the first-order language of $\mathcal{S}$ by $\mathcal{L}$.

For a tuple $\bar{x} \in X^{n}$ consider the $n \times n$ distance matrix $D_{n}(\bar{x})=d\left(x_{i}, x_{j}\right)_{i, j<n}$. We often view this matrix as a tuple in $\mathbb{R}^{n^{2}}$ with the max norm $\|\cdot\|_{\max }$. Sometimes we suppress the subscript $n$. Note that for any matrix $A \in \mathbb{Q}^{n^{2}}$ and any positive rational $p$, there is a quantifier free positive first-order formula $\phi_{A, n, p}(\bar{x})$ in the signature above expressing that $\left\|D_{n}(\bar{x})-A\right\|_{\max }<p$. 
In this paper, the main objects are computable metric spaces. Notice that, in the notations of Definition 1.3, a separable space is computable if and only if $R_{<r}\left(q_{i}, q_{k}\right)$ and $R_{>r}\left(q_{i}, q_{k}\right)$ are uniformly $\Sigma_{1}^{0}$.

Definition 2.1. Since all partial functions can be effectively listed, we obtain a uniformly computable sequence of partial computable structures $\left(M_{e}\right)_{e \in \mathbb{N}}$ so that some of these $M_{e}$ are computable structures on metric spaces: we view $M_{e}$ as a partial computable function $\psi$ such that $r_{p}=$ $\psi(i, j, p)_{p \in \mathbb{N}}$ converges rapidly (in the sense above) to $d(i, j)$. It is a $\Pi_{2}^{0}$ property of $\psi$ to be total and describe a metric space. We denote the completion of $M_{e}$ by $\operatorname{cp}\left(M_{e}\right)$.

Fact 2.2. For $\left(M, d,\left(p_{i}\right)_{i \in \mathbb{N}}\right)$ a computable metric space, and $W$ a c.e. set, $\left(p_{i}\right)_{i \in W}$ is a computable structure on the space $\left.\operatorname{cp}\left(\left(p_{i}\right)_{i \in W}\right), d\right)$.

Proof. If $W$ is infinite, we use a computable bijection $f: \omega \rightarrow W$ to define a computable structure $\left(r_{i}\right)_{i \in \mathbb{N}}$ on $\left.\operatorname{cp}\left(\left(p_{i}\right)_{i \in W}\right), d\right)$ by the rule $r_{i}=p_{f(i)}$.

Infinitary computable formulas. The language $\mathcal{L}_{\omega_{1} \omega}^{c}$ is a countable fragment of $\mathcal{L}_{\omega_{1} \omega}$. The atomic formulas are open finitary formulas in the language of metric spaces introduced above, with $\neg$ but without $=$. We allow computably enumerable conjunctions, computably enumerable disjunctions, and quantification over a variable.

In contrast to computable model theory, a computable structure on a space is not the whole space but a dense subset of it. Thus, for a computable metric structure $M_{e}$ and $\phi$ a computable infinitary formula, $\operatorname{cp}\left(M_{e}\right) \models \phi$ and $M_{e}=\phi$ have different interpretations.

The hierarchy of such formulas is defined similarly to the countable case; see the book of Ash and Knight [2]. In our specific case, the important modification is that $D_{<q}(x, y)$, for a rational $q$ and special points $x$ and $y$, should be understood as a $\Sigma_{1}$ formula, and similarly for $D_{<q}(x, y)$.

Informally, in the calculation of the complexity of a formula we also count alternations of infinitary conjunctions and disjunctions. When we count these alternations, we do not distinguish the infinitary conjunction from $\forall$, and disjunction from $\exists$. So, for example, a prefix of the form $\exists \wedge \forall \vee \exists$ will have only 3 alternations. More formally, the complexity of $\bigvee_{i} \psi_{i}$ is determined using $\inf \left\{\beta: \psi \in \Sigma_{\beta}\right\}$, and similarly for conjunctions. See [2] for formal definitions. We will omit the adjective "infinitary" when it is clear from the context.

Fact 2.3. Let $\psi$ be a computable formula of complexity $\Sigma_{n}$, where $n \in \omega$. Then the set $\left\{e: M_{e} \models \psi\right\}$ is $\Sigma_{n}^{0}$. (Similarly for $\Pi_{n}$.)

Proof. By induction on the complexity of $\psi$ we can show that, if $M_{e}$ is a (partial) computable metric structure and $M_{e} \models \psi$, then $\emptyset^{(n-1)}$ will eventually recognize it.

\section{EXISTENTIAL THEORIES AND INFINITARY FORMULAS}

\section{Theorem 3.1.}

(i) Within the class of computable Polish spaces, each compact member is uniquely described by a computable $\Pi_{3}$ axiom. 
(ii) The orbits of special elements in a compact computable metric space (under the action of its automorphism group) are given uniformly by computable $\Pi_{2}$ formulas.

Proof. We will need a result due to Friedman, Fokina, Körwien and Nies (2012) which itself is based on Gromov's work (see [11, proof of 14.2.1]).

Proposition 3.2. Let $X, Y$ be compact metric spaces. Suppose that tuples $\widetilde{a} \in X^{p}, \widetilde{b} \in Y^{p}$ satisfy the same existential positive finitary formulas. Then there is an isometry from $X$ to $Y$ mapping $\widetilde{a}$ to $\widetilde{b}$.

Proof. It is well-known that any isometric self-embedding of a compact metric space is onto (see [11, proof of 14.2.1]). Thus, it suffices to find an isometric embedding of $X$ into $Y$ mapping $\widetilde{a}$ to $\widetilde{b}$. The following lemma slightly extends the above-mentioned result of Gromov (see [11, Exercise 14.2.3]).

Lemma 3.3. Suppose that for every $\epsilon>0$, for any $n$ and tuple $\bar{x} \in X^{\bar{n}}$ there is a tuple $\bar{y} \in Y^{\bar{n}}$ such that $\|D(\widetilde{a}, \bar{x})-D(\widetilde{b}, \bar{y})\|_{\text {max }}<\epsilon$. Then there is an an isometric embedding of $X$ to $Y$ mapping $\widetilde{a}$ to $\widetilde{b}$.

It now suffices to show that if $\widetilde{a} \in X^{n}, \widetilde{b} \in Y^{n}$ satisfy the same existential positive formulas, the hypothesis of the lemma is satisfied. For every $n \times n$ rational matrix $A$, there is a formula $\phi_{A, n, \epsilon}(\bar{x})$ saying that $\left\|D_{n}(\bar{x})-A\right\|_{\max }<$ $\epsilon / 2$. Given $\bar{x} \in X^{n}$ choose a rational $(k+n) \times(k+n)$ matrix $A$ such that

$$
\|D(\widetilde{a}, \bar{x})-A\|_{\max }<\epsilon / 2 .
$$

Thus $\exists \bar{x} \phi_{A, n+k, \epsilon / 2}(\widetilde{a}, \bar{x})$ holds in $X$. Hence there is $\bar{y} \in Y^{n}$ such that $\phi_{A, n+k, \epsilon / 2}(\widetilde{b}, \bar{y})$ holds in $Y$. This implies $\|D(\widetilde{a}, \bar{x})-D(\widetilde{b}, \bar{y})\|_{\max }<\epsilon$ as required.

We prove (i) of the theorem. Note that a complete metric space is compact iff it is totally bounded, namely, satisfies the computable sentence

$$
\bigwedge_{q \in \mathbb{Q}^{+}} \bigvee_{n \in \mathbb{N}} \exists x_{0} \ldots x_{n-1} \forall y \bigvee_{i<n} d\left(x_{i}, y\right)<q
$$

We can replace each quantifier by a quantifier restricted to special points, and also replace $d\left(x_{i}, y\right)<q$ by $\neg\left(d\left(x_{i}, y\right)>q\right)$ with the meaning $d\left(x_{i}, y\right) \leq$ $q$. Let $\theta$ be the resulting computable sentence. The quantifier $\bigvee_{i<n}$ is finitary and does not contribute any extra complexity to the formula. Thus, $\theta$ is computable $\Pi_{3}$. Clearly, $M_{e} \models \theta$ if and only if $\operatorname{cp}\left(M_{e}\right) \models \theta$.

We take $M_{e}$ a computable structure on a Polish space. For the tuple $\widetilde{a}=\emptyset$ of special points we let $\psi$ be a conjunction of all formulas $\exists \bar{x} \phi_{B, k, \epsilon}(\bar{x})$ (with quantification over special points, $B$ a rational $k \times k$ matrix, $\epsilon$ a positive rational) which are true on $M_{e}$. Note that $\operatorname{cp}\left(M_{e}\right) \models \exists \bar{x} \phi_{B, k, \epsilon}(\bar{x})$ if and only if the corresponding restricted formula holds on $M_{e}$. Thus, the conjunction is in fact c.e. since we can enumerate all such sentences which are true on $M_{e}$. Therefore, $\psi$ is computable $\Pi_{2}$. The desired computable axiom is $\mathcal{F}=\theta \wedge \psi$ which is of complexity $\Pi_{3}$.

We prove (ii). The orbit of a tuple $\widetilde{a}$ of special points in a compact computable Polish space is definable by the conjunction of $\exists \bar{x} \phi_{A, n+k, \epsilon / 2}(\widetilde{a}, \bar{x})$ 
which hold on $M_{e}$. Given $\widetilde{a}$ we can effectively list all formulas $\phi_{A, n+k, \epsilon / 2}(\widetilde{a}, \bar{x})$ such that $M_{e} \models \exists \bar{x} \phi_{A, n+k, \epsilon / 2}(\widetilde{a}, \bar{x})$. Thus, the disjunction of all such formulas, with $\widetilde{a}$ replaced by a tuple of variables $\widetilde{y}$, is effective. Similarly to the proof of (1) above, we have $M_{e} \models \exists \bar{x} \phi_{A, n+k, \epsilon / 2}(\widetilde{a}, \bar{x}) \Leftrightarrow \operatorname{cp}\left(M_{e}\right) \models$ $\exists \bar{x} \phi_{A, n+k, \epsilon / 2}(\widetilde{a}, \bar{x})$, for every $\widetilde{a} \in M_{e}$ and every parameters $A, n, k$ and $\epsilon$.

\section{Descriptive Complexity of index Sets}

Recall from Definition 2.1 that $\operatorname{cp}\left(M_{e}\right)_{e \in \omega}$ is an effective listing which includes all computable metric spaces.

Fact 4.1. The set $\operatorname{Inf}=\left\{e: \operatorname{cp}\left(M_{e}\right)\right.$ is infinite $\}$ is $\Pi_{2}^{0}$-complete.

Proof. We need the approximation to the distance function to be total, this is an $\forall \exists$ property. The rest can be checked using $0^{\prime}$. For the completeness, we define a 1-reduction of the $\Pi_{2}^{0}$-complete $T o t=\left\{e: \varphi_{e}\right.$ total $\}$ to Inf by the following rule. Given $e$, if we see $\varphi_{e}(x) \downarrow$ for every $x \leq y$, we define $d(y, j)=y-j$ for every $j \leq y$. As a result, we either construct the standard computable structure on the descrete space of the natural numbers, or will be stuck at our definition of the distance function.

Proposition 4.2. (i) The set $\left\{e: \mathrm{cp}\left(M_{e}\right)\right.$ is locally compact $\}$ is $\Pi_{1}^{1}$-complete. (ii) The set $\left\{e: \operatorname{cp}\left(M_{e}\right)\right.$ is connected $\}$ is $\Pi_{1}^{1}$-hard.

Proof. A complete metric space $X$ is locally compact iff for each $x \in X$, there is rational $\epsilon>0$ such that the closed ball $K=K_{\epsilon}(x)$ is compact. If $X=$ $\operatorname{cp}\left(M_{e}\right)$, then from a Cauchy name $f$ for $x$ we can compute a presentation of $K$ as computable metric space relative to $f$, where the special points are the special points $p$ of $M_{e}$ with $d(x, p)<\epsilon$. Then by (1) and the discussion thereafter, compactness of $K$ is arithmetical in $f$. (On the other hand notice that being connected is merely $\Pi_{2}^{1}$.)

We now prove the $\Pi_{1}^{1}$-hardness. As usual let $[T]$ denote the set of infinite branches of a tree $T \subseteq \omega^{<\omega}$, and note that $[T]$, unless empty, is a metric space via $d(f, g)=2^{-k}$, where $k$ is minimal such that $f(k) \neq g(k)$. Also, $[T]$ is locally compact iff for each $f \in[T]$ there is $n$ such that $T$ with the dead ends removed is finitely branching above $f \uparrow_{n}$.

We encode the problem whether a computable tree has an infinite branch, which is well known to be $\Pi_{1}^{1}$-complete. Let

$$
F(T)=\left\{\langle\sigma, \tau\rangle: \sigma \in T \wedge \tau \in \omega^{<\omega} \wedge|\sigma|=|\tau|\right\} .
$$

Via the Cantor pairing function we can view $F(T)$ as a subtree of $\omega^{<\omega}$, and hence as a computable metric space. If $[F(T)]$ is nonempty, it is neither locally compact, nor connected. Now let $M_{T}$ be the computable metric space obtained by adjoining an isolated point at distance 2 to $[F(T)]$. Then $[T] \neq \emptyset \Leftrightarrow M_{T}$ is locally compact $\Leftrightarrow M_{T}$ is connected.

\section{Theorem 4.3.}

(i) The index set $\mathrm{CSp}$ of compact computable metric spaces is $\Pi_{3}^{0}$-complete.

(ii) The isomorphism problem for compact computable metric spaces is $\Pi_{2}^{0}$-complete within $\Pi_{3}^{0}$. 
Proof. (i) Recall that, for a sentence $\phi \in \mathcal{L}_{\omega_{1} \omega}^{c}$, the expressions $M_{e} \models \psi$ and $\operatorname{cp}\left(M_{e}\right) \models \phi$ have different interpretations: In the former we treat $\psi$ as a computable formula with quantifiers ranging over special points. In the latter $\psi$ is understood as an formula from $\mathcal{L}_{\omega_{1} \omega}$ with quantifiers ranging over the completion. We use notation from the proof of Theorem 3.1 (1). The sentence $\mathcal{F}$ has the following property. For each $e$, if $M_{e}$ is a structure on a Polish space, then

$$
M_{e}=\mathcal{F} \Leftrightarrow \operatorname{cp}\left(M_{e}\right) \mid \mathcal{F} .
$$

Thus, we have $\operatorname{CSp}=\left\{e: \operatorname{cp}\left(M_{e}\right) \models \theta\right\}=\left\{e: M_{e} \models \theta\right\}$. Now, by Facts 4.1 and 2.3, we have that CSp is $\Pi_{3}^{0}$.

We now prove $\Pi_{3}^{0}$-completeness of CSp. The standard computable structure on Baire space $\omega^{\omega}$ is given by the collection of finite strings of natural numbers. We fix a $\Pi_{3}^{0}$-complete set $S$ and a computable predicate $P$ such that $x \in S \Leftrightarrow \forall y \exists \exists^{<\infty} z P(x, y, z)$. By Fact 2.2, it is sufficient to construct a uniformly c.e. family $\left(C_{x}\right)_{x \in \mathbb{N}}$ of substructures of the standard structure on $\omega^{\omega}$ which satisfies $x \in S \Leftrightarrow \operatorname{cp}\left(C_{x}\right)$ is compact. By uniformity, there will exist a total computable $f$ such that $C_{x}=M_{f(x)}$ witnessing the desired reduction.

Construction. At stage -1, enumerate $01^{y}$ into the structure $C_{x}$ for every $y$. At stage $s \geq 0$, we enumerate $01^{y} z$ with $z \leq s$ into $C_{x}$ if $P(x, y, z)$ holds.

If $x \in S$ then each of the $01^{y}$ will have only finitely many extending strings, and the space $\operatorname{cp}\left(C_{x}\right)$ is compact. If $x \notin S$, then there is at least one string $01^{y}$ witnessing that $\operatorname{cp}\left(C_{x}\right)$ is not compact.

Remark 4.4. It follows from the $\Pi_{3}^{0}$-completeness of CSp and Fact 2.3 that the complexity of the sentence $\mathcal{F}$ from Theorem 3.1 can not be reduced.

(ii) Given $e, j \in \mathrm{CSp}$, we can effectively produce a computable $\Pi_{2}$ formula $\psi$ in the notation of Theorem 3.1(1) which completely describes the isomorphism type of $M_{j}$. To see if $\mathrm{cp}\left(M_{e}\right) \cong \mathrm{cp}\left(M_{j}\right)$ it suffices to check if $M_{e} \models \psi$. By Fact $2.3, \Psi_{j}=\left\{i: M_{i} \models \psi\right\}$ is $\Pi_{2}^{0}$, and it is actually uniformly $\Pi_{2}$ in the index of the formula $\psi$. Thus, the condition $e \in \Psi_{j}$ is $\Pi_{2}^{0}$ uniformly in $e$ and $j$.

For the completeness, fix a $\Pi_{2}^{0}$-complete set $S$ and a computable binary predicate $R$ such that $x \in S \Leftrightarrow \exists^{\infty} y R(x, y)$. Let $j$ be any computable index of the standard structure on Cantor space. For every $x$, we construct a c.e. closed subspace $C_{x}$ of the standard structure on Cantor space. By Fact 2.2, we will get a computable structure on a compact space.

In the construction, if we see another $y$ for which $R(x, y)$ holds, we enumerate finite strings of length $\leq y$ from the standard structure into $C_{x}$. As a result, we will have $C_{x}$ isomorphic to the whole Cantor space if, and only if, $x \in S$. Let $f$ be a total computable function such that $C_{x}=M_{f(x)}$. We have $M_{j} \cong M_{f(x)}$ if and only if $x \in S$, as desired.

Next we study the complexity of whether a computable metric space is a continuum.

Proposition 4.5. The index set CCSp of compact and connected computable metric spaces is $\Pi_{3}^{0}$-complete. 
Proof. Note that a metric space $X$ is connected iff for each nonempty open sets $U, V$, we have $C=X-(U \cup V)=\emptyset \Rightarrow U \cap V \neq \emptyset$. Suppose now we are given a compact computable metric space $X=\mathrm{cp}\left(M_{e}\right)$. For connectedness, we may restrict $U, V$ to finite unions of basic open sets of the form $B_{\epsilon}(p)$ where $\epsilon \in \mathbb{Q}^{+}$and $p$ is a special point. We may effectively in $e$ obtain a $\emptyset^{\prime}$-computable map $g$ from $2^{\omega}$ onto $X$. Thus $C=\emptyset$ is equivalent to $g^{-1}(C)=\emptyset$. Since the latter is a $\Pi_{1}^{0}\left(\emptyset^{\prime}\right)$ class, this condition is $\Sigma_{2}^{0}$. The condition $U \cap V \neq \emptyset$ is $\Sigma_{1}^{0}$ since this set contains a special point unless empty. Thus being connected is in fact $\Pi_{2}^{0}$ within the $\Pi_{3}^{0}$ set CSp.

Let $S$ be any $\Pi_{3}^{0}$-complete set, and choose a uniformly c.e. double sequence $\left(V_{i, n}\right)$ of initial segments of $\omega$ such that $i \in S \leftrightarrow \forall n V_{i, n} \neq \omega$. Let $a_{k}=1-2^{-k}$. Given $i$, we can compute an index $e$ for the computable metric space the Cartesian product $\Pi_{n \in \omega}\left[0, a_{\left|V_{i, n}\right|}\right]$ with the canonical computable structure obtained from the enumerations of the $V_{i, n}$, and the metric inherited from the standard metric on the Hilbert cube $[0,1]^{\omega}$. Clearly $M_{e}$ is connected, and $M_{e}$ is compact iff $i \in S$.

\section{5. $\Delta_{3}^{0}$ CATEGORICITY}

Definition 5.1. Let $S \subseteq \omega$ be an oracle. An isometry $\Phi$ from a computable metric space $\left(X, d,\left(q_{i}\right)_{i \in \mathbb{N}}\right)$ to a computable metric space

$\left(Y, d,\left(p_{i}\right)_{i \in \mathbb{N}}\right)$ is computable in $S$ if there is a Turing machine with oracle $S$ which, on inputs $i, k$, outputs the $k$-th term of a Cauchy name for $\Phi\left(q_{i}\right)$.

We say that a computable metric space is $\Delta_{n}^{0}$-categorical if between each of its computable presentations, there is an isometry computable relative to $\emptyset^{(n-1)}$.

Theorem 5.2. Each compact computable metric space is $\Delta_{3}^{0}$ categorical.

Proof. Let $\mathcal{X}=\left(X, d,\left(p_{i}\right)_{i \in \mathbb{N}}\right)$ and $\mathcal{Y}=\left(Y, d,\left(q_{j}\right)_{j \in \mathbb{N}}\right)$ be compact computable metric spaces. Suppose that $\mathcal{X}$ can be isometrically embedded into $\mathcal{Y}$. We show that then there is a $\Delta_{3}^{0}$ embedding; this is sufficient by symmetry.

Recall distance matrices $D_{n}$ from Section 2. Let $\epsilon_{n}=2^{-n}$. There is a computable array of special points $\left\langle y_{i}^{n}\right\rangle_{i<n}$ in $Y$ such that, where $\bar{y}^{n}=$ $\left\langle y_{0}^{n}, \ldots, y_{n-1}^{n}\right\rangle$, we have

$$
\left\|D_{n}\left(\left\langle p_{0}, \ldots, p_{n-1}\right\rangle\right)-D_{n}\left(\bar{y}^{n}\right)\right\|_{\max }<\epsilon_{n} .
$$

We define a $\emptyset^{\prime \prime}$ computable array of special points $\left\langle w_{i}^{n}\right\rangle_{i \leq n, 0<n}$ in $Y$ such that for each $n$, where $\bar{w}^{n}=\left\langle w_{0}^{n}, \ldots, w_{n-1}^{n}\right\rangle$, we have

$$
\left|\left\{k>n: d\left(y^{k} \uparrow_{n}, \bar{w}^{n}\right)<\epsilon_{n}\right\}\right|=\infty .
$$

For each $n \geq 1$, the space $Y^{n}$ is equipped with the maximum metric. We use compactness of the $Y^{n}$ throughout. Let $w_{0}^{1} \in Y$ be a special point such that $A_{1}=\left\{k: d\left(y_{0}^{k}, w_{0}^{1}\right)<\epsilon_{1}\right\}$ is infinite. Then (2) holds for $n=1$.

(a) Increasing the dimension. Let $w_{1}^{1}$ be a special point in $Y$ such that $C_{1}=\left\{k \in A_{1}: d\left(y_{1}^{k}, w_{1}^{1}\right)<\epsilon_{1}\right\}$ is infinite.

(b) Refining the sequence. Let $\bar{w}^{2} \in Y^{2}$ be a special point in $B_{\epsilon_{1}}\left(\left\langle w_{0}^{1}, w_{1}^{1}\right\rangle\right)$ such that $A_{2}=\left\{k \in B_{1}: d\left(\bar{y}^{k} \uparrow_{2}, \bar{w}^{2}\right)<\epsilon_{2}\right\}$ is infinite.

We continue this process. Suppose $\bar{w}^{n}$ (and hence $A_{n}$ ) has been defined 
(a) Let $w_{n}^{n}$ be a special point in $Y$ such that

$$
C_{n}=\left\{k \in A_{n}: k>n \wedge d\left(y_{n}^{k}, w_{n}^{n}\right)<\epsilon_{n}\right\}
$$

is infinite.

(b) Let $\bar{w}^{n+1} \in Y^{n+1}$ be a special point in $B_{\epsilon_{n}}\left(\left(\bar{w}^{n}\right)^{\wedge} w_{n}^{n}\right)$ such that

$$
A_{n+1}=\left\{k \in C_{n}: d\left(\bar{y}^{k} \uparrow_{n+1}, \bar{w}^{n+1}\right)<\epsilon_{n+1}\right\}
$$

is infinite. Then (2) holds for $n+1$.

Note that the sequence $\left\langle w_{i}^{n}\right\rangle_{i<n, 0<n}$ is indeed $\emptyset^{\prime \prime}$-computable because we uniformly in the previously defined special points obtain indices for the potential c.e. sets $C_{n}, A_{n+1}$. It takes $\emptyset^{\prime \prime}$ as an oracle to pick the next special points in such a way that the relevant set is infinite. Also note that $d\left(w_{r}^{n}, w_{r}^{n+1}\right)<\epsilon_{n}$ for each $n>r$. Thus, the sequence of points in $Y$ $z_{r}=\lim _{n>r} w_{r}^{n}$ is computable in $\emptyset^{\prime \prime}$. It now suffices to show that the map $x_{i} \mapsto z_{i}$ preserves distances. Let $i<j$. Given $n$, by (2) pick $k>n$ such that $d\left(y^{k} \uparrow_{n}, \bar{w}^{n}\right)<\epsilon_{n}$. Then, by the definitions,

$$
\begin{aligned}
\left|d\left(z_{i}, z_{j}\right)-d\left(w_{i}^{n}, w_{j}^{n}\right)\right| & \leq 2 \epsilon_{n} \\
\left|d\left(w_{i}^{n}, w_{j}^{n}\right)-d\left(y_{i}^{k}, y_{j}^{k}\right)\right| & \leq \epsilon_{n} \\
\left|d\left(y_{i}^{k}, y_{j}^{k}\right)-d\left(x_{i}, x_{j}\right)\right| & \leq \epsilon_{n} .
\end{aligned}
$$

Therefore, $\left|d\left(z_{i}, z_{j}\right)-d\left(x_{i}, x_{j}\right)\right| \leq 4 \epsilon_{n}$.

The bound on the complexity of an isomorphism we obtained in Theorem 5.2 is not optimal. We can prove the following strengthening saying that some isomorphism is low relative to $\emptyset^{\prime}$.

Theorem 5.3. Let $\mathcal{X}=\left(X, d,\left(p_{i}\right)_{i \in \mathbb{N}}\right)$ and $\mathcal{Y}=\left(Y, d,\left(q_{j}\right)_{j \in \mathbb{N}}\right)$ be isometric compact computable metric spaces. Then there is a function $h$ on $\mathbb{N}$ with $h^{\prime} \leq_{T} \emptyset^{\prime \prime}$ which computes an isometry.

Proof. The proof is an extension of the previous argument. We build a nonempty $\Pi_{1}^{0}\left(\emptyset^{\prime}\right)$ class $\mathcal{D}$ of isometric embeddings from $\mathcal{X}$ to $\mathcal{Y}$, and then apply symmetry to obtain an isometry. The class $\mathcal{D}$ consists of the branches of a tree of special points. Since the space is compact, the level size of the tree is bounded by a $\emptyset^{\prime}$-computable function $f$. Then, by the low basis theorem relative to $\emptyset^{\prime}$, we obtain an isometric embedding as required.

We give the details. For each $n$, we identify a tuple $\left\langle i_{0}, \ldots, i_{n-1}\right\rangle$ in $\mathbb{N}^{n}$ with the special point $\left\langle q_{i_{0}}, \ldots, q_{i_{n-1}}\right\rangle$ of $Y^{n}$. We introduce a basic tree that consists of strings $\alpha$ such that $\alpha(2 n)$ is a special point of $Y$, and for $n>0$, $\alpha(2 n-1)$ is a special point of $Y^{n}$. Via the usual computable bijections of $\mathbb{N}^{k}$ and $\mathbb{N}$, we can identify this basic tree in an effective way with $\omega^{<\omega}$.

Since $Y$ is compact, there is a $\emptyset^{\prime}$-computable function $f$ such that for each $n$, we have

$$
Y=\bigcup_{p<f(2 n)} B_{\epsilon_{n}}(p)
$$

and for each $\bar{q}<f(2 n-1)$ and $p<f(2 n)$,

$$
B_{\epsilon_{n}}(\langle\bar{q}, p\rangle) \subseteq \bigcup_{\widetilde{r}<f(2 n+1)} B_{\epsilon_{n+1}}(\widetilde{r}) .
$$


(Note that the number $\bar{q}$ is thought of as a special point of $Y^{n}$, and $\langle\bar{q}, p\rangle$ and $\widetilde{r}$ are thought of as special points of $Y^{n+1}$.)

Recall the computable array of special points $\left\langle y_{i}^{n}\right\rangle_{i<n}$ in $Y$. Let $A_{\emptyset}=\mathbb{N}$. For a string $\sigma=\tau^{\wedge} p$ where $p \in \omega$ and $\tau$ has even length, let

$$
C_{\sigma}=\left\{k \in A_{\tau}: d\left(\bar{y}^{k} \uparrow_{n+1}, p\right)<\epsilon_{n+1}\right\} .
$$

For a string $\eta=\sigma^{\wedge} \tilde{r}$ where $\tilde{r} \in \omega$ and $\sigma$ has odd length, let

$$
A_{\eta}=\left\{k \in C_{\sigma}: d\left(\bar{y}^{k} \uparrow_{n+1}, \tilde{r}\right)<\epsilon_{n+1}\right\} .
$$

Now consider the $\Pi_{1}^{0}\left(\emptyset^{\prime}\right)$ subclass of $\omega^{\omega}$

$$
\mathcal{D}=\left\{h \leq f: \forall n\left[\left|C_{\left.h\right|_{2 n+1}}\right|=\infty \wedge\left|A_{\left.h\right|_{2 n}}\right|=\infty\right\} .\right.
$$

By the choice of $f$ we have $\mathcal{D} \neq \emptyset$. From any $h$ in this class we compute a double sequence $\left\langle w_{i}^{n}\right\rangle_{i<n, 0<n}$ by letting $w_{i}^{n}$ be the $i$-th component of $h(2 n)$. So $z_{r}=\lim _{n>r} w_{r}^{n}$ is computable in $h$. The map $x_{i} \mapsto z_{i}$ preserves distances. Since $\mathcal{D}$ is bounded by the $\emptyset^{\prime}$-computable function $f$, by the Low Basis Theorem relative to $\emptyset^{\prime}$ there is $h \in \mathcal{D}$ with $h^{\prime} \leq_{\mathrm{T}} \emptyset^{\prime \prime}$ as required.

Theorem 5.2 can not be improved to $\Delta_{2}^{0}$-categoricity. In the theorem below we are building a c.e. substructure of the standard structure on Cantor space (recall Fact 2.2). In the proof, we will identify special points with their respective numbers. To make the space corresponding to our structure not $\Delta_{2}^{0}$-categorical, we use the following observation:

Fact 5.4. Let $U:\left(M_{1}, d_{1},\left(p_{i}\right)_{i \in \mathbb{N}}\right) \rightarrow\left(M_{2}, d_{2},\left(u_{j}\right)_{j \in \mathbb{N}}\right)$ be a $\Delta_{2}^{0}$ map between computable metric spaces. Then there exists a computable function $f$ of three arguments such that for every $i$,

$$
U\left(p_{i}\right)=\lim _{n}\left(\lim _{s} f(i, n, s)\right),
$$

where $(f(i, n, s))_{s \in \mathbb{N}}$ stabilizes for every $i, n$, and additionally $\left(\lim _{s} f(i, n, s)\right)_{n \in \mathbb{N}}$ is a Cauchy name.

Proof. By assumption, there exists a $0^{\prime}$-computable function $g$ such that $U\left(p_{i}\right)=\lim _{n} u_{g(i, n)}$, and $\left(u_{g(i, n)}\right)_{n \in \mathbb{N}}$ is a Cauchy name. By the Limit Lemma [20], there exists a total computable function $f$ such that $g(i, n)=$ $\lim _{s} f(i, n, s)$, and the limit here means that the sequence

$$
f(i, n, 0), f(i, n, 1), \ldots
$$

stabilizes from some $k$ on.

Theorem 5.5. There exists a compact computable metric subspace which is not $\Delta_{2}^{0}$-categorical.

Proof idea. We construct isometric c.e. subspaces, $L$ and $R$, of Cantor space. The $e^{\prime} t h$ potential $\Delta_{2}^{0}$ map between $L$ and $R$ will be associated with a finite string $\sigma_{e}$ in $L$. The string $\sigma_{e}$ will be definable in $L$ in the language of metric spaces. In the following, we may think of both $L$ and $R$ as of collections of finite binary strings. At every stage we will have a finite tree of strings. Using the finite strings extending the witness string $\sigma_{e}$, we will "grow" two potentially infinite extensions of $\sigma_{e}$. The $e^{\prime} t h$ potential map has to choose how these extensions are mapped to the corresponding locations 
in $R$ extending the uniquely defined isometric image of $\sigma_{e}$. If the $e^{\prime} t h$ map stabilizes, we create extensions of $\sigma_{e}$ of different length so that the image via the $e^{\prime} t h$ potential map gives a wrong length in $R$. If the map does not stabilize, we grow the extensions to infinity. In both cases the corresponding locations in $L$ and $R$ are isometric, but the $e^{\prime} t h$ potential map is defeated.

Proof. We will be enumerating two computable structures, $L=\left(\alpha_{i}\right)_{i \in \mathbb{N}}$ and $R=\left(\beta_{j}\right)_{j \in \mathbb{N}}$, on a closed subspace of Cantor space. Each of the $\alpha_{i}$ and $\beta_{j}$ correspond to a finite string from the standard computable structure on Cantor space. In the following, we identify special points, their respective numbers, and the corresponding finite strings. We may also identify finite strings $\xi$ and $\xi\left(0^{n}\right)$ since they correspond to the same element $\xi\left(0^{\infty}\right)$.)

We need to make the closures of $L$ and $R$ isometric but not $\Delta_{2}^{0}$-isometric. It follows from Fact 5.4 that it is sufficient to meet, for every $e$, the requirement:

$$
P_{e}: \lim _{n}\left(\lim _{s} \varphi_{e}(i, n, s)\right) \text { total } \Rightarrow \lim _{n}\left(\lim _{s} \varphi_{e}(i, n, s)\right) \text { is not an isometry, }
$$

where the first limit is taken in $R$ with respect to the metric inherited from Cantor space, and the second means that the sequence $\left(\varphi_{e}(i, n, s)\right)_{s \in \mathbb{N}}$ stabilizes.

For every $e \geq 0, P_{e}$ will have witnesses strings $\sigma_{e} \in L$ and $\tau_{e} \in R$. More specifically, we set $\sigma_{e}=\tau_{e}=0^{e} 1$. At later stages, $P_{e}$ will possibly enumerate only strings extending $\sigma_{e}$ or $\tau_{e}$ (in $L$ and $R$, respectively).

The strategy for $P_{e}$. The strategy immediately enumerates $\sigma_{e}$ and $\sigma_{e} 1$ into $L$, and also enumerates $\tau_{e}$ and $\tau_{e} 1$ into $R$. Then the strategy performs the substages below.

(1) Wait for $\varphi_{e}\left(\sigma_{e}, 2 e+2, s\right)$ to converge to (the index of) a string $\rho_{s} \supseteq \tau_{e}$ at stage $s$. If we ever see such a computation, then let $j_{s} \in\{0,1\}$ be such that $\tau_{e} j_{s} \subseteq \rho_{s}$. (Recall that we identify $\tau_{e}$ and $\tau_{e} 0$.)

(2) For every $t \leq s$ enumerate $\sigma_{e} 01^{t}$ into $L$ and $\tau_{e}\left(1-j_{s}\right) 1^{t}$ into $R$.

We repeat the process above. We wait for $s^{\prime}>s$ and a computation $\varphi_{e}\left(\sigma_{e}, e+2, s^{\prime}\right)=\rho_{s^{\prime}} \supseteq \tau_{e}$ with $j_{s^{\prime}} \neq j_{s}$. If we ever see such a computation, then for every $t \leq s$ we enumerate $\sigma_{e} 1^{t+1}$ into $L$ and $\tau_{e} j_{s} 1^{t}$ into $R$, and then go to (2) with $s^{\prime}$ in place of $s$.

Construction. At stage $s \geq 0$, let $P_{e}, e \leq s$, act according to their instructions.

Verification. The strategies altogether will possibly grow only extensions of $\sigma_{e}$ and $\tau_{e}$. Notice that $\emptyset$ (identified with $0^{\infty}$ ) is the only point in the completion of $L$ having Cantor-Bendixson rank 2. The same can be said about $R$. The points $x$ extending $\sigma_{e}$ satisfy the formula $d(\emptyset, x)=2^{-e}$ in the completion of $L$, and the points $y$ extending $\tau_{e}$ satisfy the same formula in the completion of $R$. Since the formulae are different for different $e$, we conclude that an isometry from $L$ to $R$ (if it exists) should map an element extending $\sigma_{e}$ into an element extending $\tau_{e}$.

We have the following cases: 
Case 1. Either $\varphi_{e}\left(\sigma_{e}, e+2, s\right)$ diverges for some $s$, or eventually settles on $\xi$ which does not extend $\tau_{e}$. In the latter case $d\left(\xi, \tau_{e}\right)>2^{-e-1}$, contradicting the choice of $\tau_{e}$ and $\sigma_{e}$.

Case 2. The sequence $\left(\varphi_{e}\left(\sigma_{e}, e+2, s\right)\right)_{s \in \mathbb{N}}$ stabilizes on $\rho \supset \tau_{e} j$, where $j=\lim _{s} j_{s}$. In this case the strategy guarantees that no isometry can possibly map strings extending $\sigma_{e} 0$ to strings extending $\tau_{e} j$. (All strings extending $\sigma_{e} 0$ and $\tau_{e} j$ correspond to isolated points in $L$ and $R$, respectively. The number of isolated strings extending $\sigma_{e} 0$ is not the same as the number of those extending $\tau_{e} j$, this property can be recognized by a formula.)

Case 3. The sequence $\left(\varphi_{e}\left(\sigma_{e}, e+2, s\right)\right)_{s \in \mathbb{N}}$ never stabilizes. In this case the strategy will possibly enumerate all strings of the form $\sigma_{e} v 1^{k}$ into $L$ and $\tau_{e} w 1^{k}$ into $R$, where $v, w \in\{0,1\}$.

In each case $P_{e}$ is met. Notice that in each of the three cases the fragment of $L$ containing extensions of $\sigma_{e}$ will be isometric to the corresponding fragment of $R$ containing extensions of $\tau_{e}$. Consequently, the definitions of $L$ and $R$ guarantee that, in fact, $L$ and $R$ are isometric (thus, their completions are isometric as well).

\section{REFERENCES}

[1] C. J. Ash. Stability of recursive structures in arithmetical degrees. Ann. Pure Appl. Logic, 32(2):113-135, 1986. 2

[2] C. J. Ash and J. Knight. Computable structures and the hyperarithmetical hierarchy, volume 144 of Studies in Logic and the Foundations of Mathematics. North-Holland Publishing Co., Amsterdam, 2000. 1, 2, 3, 5

[3] C.J. Ash. Recursive labeling systems and stability of recursive structures in hyperarithmetical degrees. Trans. Amer. Math. Soc., 298:497-514, 1986. 3

[4] Ewan J. Barker. Back and forth relations for reduced abelian p-groups. Ann. Pure Appl. Logic, 75(3):223-249, 1995. 3

[5] Vasco Brattka, Peter Hertling, and Klaus Weihrauch. A tutorial on computable analysis. In New computational paradigms, pages 425-491. Springer, New York, 2008. 1, 3

[6] Wesley Calvert and Julia F. Knight. Classification from a computable viewpoint. Bull. Symbolic Logic, 12(2):191-218, 2006. 2

[7] Barbara F. Csima, Antonio Montalbán, and Richard A. Shore. Boolean algebras, Tarski invariants, and index sets. Notre Dame J. Formal Logic, 47(1):1-23, 2006. 2

[8] Rod Downey and A. Melnikov. Effectively categorical abelian groups. To appear. 3

[9] Yuri L. Ershov and S. S. Goncharov. Constructive models. Siberian School of Algebra and Logic. Consultants Bureau, New York, 2000. 1

[10] A. Fröhlich and J. C. Shepherdson. Effective procedures in field theory. Philos. Trans. Roy. Soc. London. Ser. A., 248:407-432, 1956. 3

[11] Su Gao. Invariant descriptive set theory, volume 293 of Pure and Applied Mathematics (Boca Raton). CRC Press, Boca Raton, FL, 2009. 1, 6

[12] S. S. Goncharov and Dzh. Naĭt. Computable structure and antistructure theorems. Algebra Logika, 41(6):639-681, 757, 2002. 2

[13] Bakhadyr Khoussainov, André Nies, Sasha Rubin, and Frank Stephan. Automatic structures: richness and limitations. Log. Methods Comput. Sci., 3(2):2:2, 18, 2007. 2

[14] A. I. Mal'cev. On recursive Abelian groups. Dokl. Akad. Nauk SSSR, 146:1009-1012, 1962. 3

[15] Charles F. D. McCoy. $\Delta_{2}^{0}$-categoricity in Boolean algebras and linear orderings. Ann. Pure Appl. Logic, 119(1-3):85-120, 2003. 3

[16] A. Melnikov. Computably isometric spaces. To appear. 1, 4 
[17] A. Melnikov. Computable ordered abelian groups and fields. In Programs, proofs, processes, volume 6158 of Lecture Notes in Comput. Sci., pages 321-330. Springer, Berlin, 2010. 3

[18] Marian B. Pour-El and J. Ian Richards. Computability in analysis and physics. Perspectives in Mathematical Logic. Springer-Verlag, Berlin, 1989. 1, 3

[19] Michael O. Rabin. Computable algebra, general theory and theory of computable fields. Trans. Amer. Math. Soc., 95:341-360, 1960. 3

[20] Robert I. Soare. Recursively enumerable sets and degrees. Perspectives in Mathematical Logic. Springer-Verlag, Berlin, 1987. A study of computable functions and computably generated sets. 11

[21] Alan M. Turing. On computable numbers, with an application to the entscheidungsproblem. Proceedings of the London Mathematical Society, 42:230-265, 1936. 3

[22] Alan M. Turing. On computable numbers, with an application to the entscheidungsproblem. A correction. Proceedings of the London Mathematical Society, 43:544546, 1937. 3

[23] M. Vanden Boom. The effective Borel hierarchy. Fund. Math., 195(3):269-289, 2007. 2

[24] Klaus Weihrauch. Computable analysis. Texts in Theoretical Computer Science. An EATCS Series. Springer-Verlag, Berlin, 2000. An introduction. 1 